\title{
ATTAINABLE ELECTRON ENERGY IN DIODE WITH PLASMA CATHODE AT THE GIVEN VOLTAGE
}

\author{
V. Ostroushko, A. Pashchenko, I. Pashchenko \\ National Science Center “Kharkov Institute of Physics and Technology”, Kharkiv, Ukraine \\ E-mail: ostroushko-v@kipt.kharkov.ua
}

It is considered an acceleration of electrons in a one-dimensional interval, part of which is filled by ions initially compensated by electrons. For the stages of forward motion and backward motion of a part of electrons, the problem is reduced to a numerical solution of ordinary differential equations for some set of time-dependent quantities. The ratio of attainable energy to the energy corresponding to the voltage is maximum and near to 1.87475 for relatively small values of the width of the space without ions when applying a certain voltage, which, as the width is reduced, has to be reduced as a width cube.

PACS: 41.85.Ar

\section{INTRODUCTION}

When large amount of electrons accelerates in diode the part of them can obtain the energy greater then one corresponding to the applied voltage. Such effect is shown in the paper [1] for the diode with plasma cathode. The question arises about the attainable value of the ratio $\eta$ of the maximum energy obtained by some electrons to the product of elementary charge by voltage. This ratio may be called the coefficient of the energy increase. It is always greater than 1 , because electrons in diode increase the acceleration of the electrons ahead of themselves and decrease the acceleration of the electrons behind, in comparing with acceleration of single electron under the given voltage. In absence of the external circuit, the discharge of cathode and the movement of electrons to anode are accompanied with decrease of the potential difference between electrodes, though the electric field strength near anode remains constant up to going out of the first electrons to anode. And vice versa, the keeping up of the potential difference through the external circuit leads to the field strength increase in the leading point of electron flow. In the model somewhat different from one considered in [1], it may be formally obtained an infinite value of the mentioned coefficient. Namely, let the plane anode is immobilized and the infinitely thin plane cathode is accelerated to anode under the force corresponding to the electric field strength in the cathode-anode gap. To keep the voltage $U$ when the distance $x$ between electrodes is decreased and the capacity depends on $x$ as $1 / x$, one has to ensure the increase of electrode charges as $1 / x$, and then the electric field strength, which is equal to $U / x$, gives the acceleration force increase as $1 / x^{2}$, and the cathode kinetic energy increase as const $+1 / x$. But in the diode with the plasma cathode, the space filled by electrons is expanded, and the ratio $\eta$ is finite. In the present work the search for the maximum attainable value of $\eta$ in the frames of the model considered in [1] is carried out.

\section{PROBLEM FORMULATION}

The considered one-dimensional structure between cathode and anode consists of a few layers. The nearcathode interval is filled by the immobilized positive ions with the uniform density ('ion space' below, hori- zontal lines in the Fig. 1), and the near-anode interval is free from ions ('vacuum space'). At the initial time instant (see Fig. 1,a), at the motion start, the ion space is also filled by the electrons (vertical lines), which have the same density and zero velocity. It is convenient to take the distance between cathode and anode and the reciprocal plasma frequency determined by the mentioned uniform density for the units of distance and time, respectively, and to use the relevant dimensionless variables and quantities. Below, the letters $x, b$, and $\zeta$ are used for coordinates, $t$ and $\tau$ are used for time, $v$ is velocity, $n$ is electron density, $E$ is the electric field strength (more precisely, the acceleration of the elementary positive charge with electron mass in the field with the corresponding strength), $\varphi$ and $U$ are used for potential and voltage.

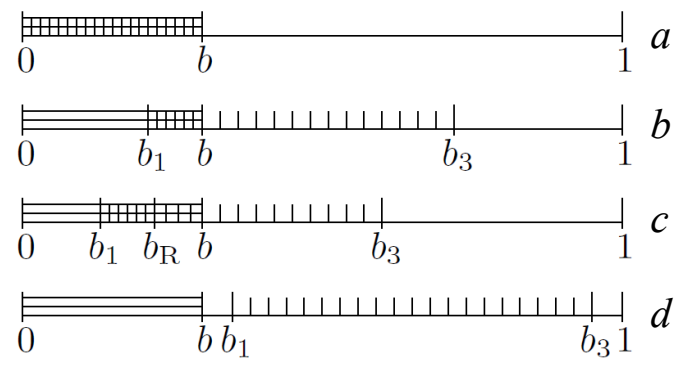

Fig. 1. Ion and electron disposition, initial and at some stages of electron motion

The electron motion at $t>0$ obeys to the equations

$$
\begin{gathered}
\partial_{t} x_{\mathrm{e}}(\zeta, t)=v_{\mathrm{e}}(\zeta, t), \\
\partial_{t} v_{\mathrm{e}}(\zeta, t)=-E_{\mathrm{e}}(\zeta, t) .
\end{gathered}
$$

Here $\partial$ is derivative, its index indicates the variable, with respect to which the derivative is taken, $x_{\mathrm{e}}(\zeta, t)$ and $v_{\mathrm{e}}(\zeta, t)$ are, respectively, coordinate and velocity at the time $t$ of the electron, which has the coordinate $\zeta$ at $t=0, E_{\mathrm{e}}(\zeta, t)$ is the strength in the point $x=x_{\mathrm{e}}(\zeta, t)$ at the time $t$. The variables $(\zeta, t)$ are Lagrange ones. Also, it is expedient to use the function $n_{\mathrm{e}}(\zeta, t)$ for the electron density in the point $x=x_{\mathrm{e}}(\zeta, t)$ at the time $t$ and the functions $v(x, t), n(x, t)$, and $E(x, t)$, for the electron velocity and density, and the field strength, at the time $t$ in the point $x$. The variable $x$ may be considered as Euler one. If $x=x_{\mathrm{e}}(\zeta, t)$ then $f(x, t)=f_{\mathrm{e}}(\zeta, t)$, where $f$ stands for $v, n$, or $E$. The 
functions $v(x, t), n(x, t)$, and $E(x, t)$ should be continuous. The initial conditions for the equations (1) and (2) are

$$
x_{\mathrm{e}}(\zeta, 0)=\zeta, v_{\mathrm{e}}(\zeta, 0)=0 .
$$

The potential counted off from the cathode potential,

$$
\varphi(x, t)=-\int_{0}^{x} E\left(x^{\prime}, t\right) d x^{\prime},
$$

at the anode has to be equal to the applied voltage value, which is assumed constant,

$$
\varphi(1, t)=U
$$

\section{INITIAL STAGE OF MOTION}

At first, it should be considered the initial stage, when all electrons are moving to anode, the inequality $\zeta^{\prime \prime}>\zeta^{\prime}$ implies the inequality $x_{\mathrm{e}}\left(\zeta^{\prime \prime}, t\right)>x_{\mathrm{e}}\left(\zeta^{\prime}, t\right)$, and the boundary coordinates of the electron space, $b_{1}(t)=x_{\mathrm{e}}(0, t), b_{3}(t)=x_{\mathrm{e}}(b, t)$, obey to the inequalities $0<b_{1}(t)<b<b_{3}(t)<1$ (Fig. 1,b).

The strength derivative in the different spatial intervals obeys to the equations

$$
\begin{aligned}
& \partial_{x} E(x, t)=1\left(0<x<b_{1}(t)\right), \\
& \partial_{\zeta} E_{\mathrm{e}}(\zeta, t)=\left[1-n_{\mathrm{e}}(\zeta, t)\right] D_{\mathrm{e}}(\zeta, t) . \\
& \left(b_{1}(t)<x_{\mathrm{e}}(\zeta, t)<b\right), \\
& \partial_{\zeta} E_{\mathrm{e}}(\zeta, t)=-n_{\mathrm{e}}(\zeta, t) D_{\mathrm{e}}(\zeta, t) . \\
& \left(b<x_{\mathrm{e}}(\zeta, t)<b_{3}(t)\right), \\
& \partial_{x} E(x, t)=0\left(b_{3}(t)<x<1\right),
\end{aligned}
$$

where $D_{\mathrm{e}}(\zeta, t)=\partial_{\zeta} x_{\mathrm{e}}(\zeta, t)$.

Let $\zeta_{\mathrm{e}}(\tau)$ be the initial coordinate of the electron, which passes the boundary $b$ at the time $\tau$, and $\tau_{\mathrm{e}}(\zeta)$ be the time, at which the electron with the initial coordinate $\zeta$ passes the boundary $b$, and so, $x_{\mathrm{e}}\left(\zeta_{\mathrm{e}}(\tau), \tau\right)=b, \quad x_{\mathrm{e}}\left(\zeta, \tau_{\mathrm{e}}(\zeta)\right)=b, \quad \tau_{\mathrm{e}}\left(\zeta_{\mathrm{e}}(\tau)\right)=\tau$, $\zeta_{\mathrm{e}}\left(\tau_{\mathrm{e}}(\zeta)\right)=\zeta$. During the considered motion stage, the condition $x_{\mathrm{e}}(\zeta, t) \in\left(b_{1}(t), b\right)$ implies the conditions $\zeta \in\left(0, \zeta_{\mathrm{e}}(t)\right)$ and $t \in\left(0, \tau_{\mathrm{e}}(\zeta)\right)$, and the condition $x_{\mathrm{e}}(\zeta, t) \in\left(b, b_{3}(t)\right)$ implies the conditions $\zeta \in\left(\zeta_{\mathrm{e}}(t), b\right)$ and $\tau_{\mathrm{e}}(\zeta)<t$. For the electron density, the continuity equation in Lagrange variables has the form

$$
\partial_{t}\left[n_{\mathrm{e}}(\zeta, t) D_{\mathrm{e}}(\zeta, t)\right]=0 \quad(0<\zeta<b) .
$$

As $x_{\mathrm{e}}(\zeta, 0)=\zeta$, the integration of (10) gives

$$
n_{\mathrm{e}}(\zeta, t) D_{\mathrm{e}}(\zeta, t)=1(0<\zeta<b),
$$

and the equalities $(7)$ and $(8)$ take the form

$$
\begin{aligned}
& \partial_{\zeta} E_{\mathrm{e}}(\zeta, t)=D_{\mathrm{e}}(\zeta, t)-1\left(0<\zeta<\zeta_{\mathrm{e}}(t)\right), \\
& \partial_{\zeta} E_{\mathrm{e}}(\zeta, t)=-1\left(\zeta_{\mathrm{e}}(t)<\zeta<b\right) .
\end{aligned}
$$

From (6), (9), (11), and (12) it follows that for the given electron distribution, the field strength values in the different points are connected with the equation

$$
E(x, t)-E(0, t)=\min (x, b)-\int d \zeta,
$$

with the integral taken over such $\zeta$, for which $x_{\mathrm{e}}(\zeta, t)<x$.

In the interval $x \in\left(b_{1}(t), b\right)$, from (1), (2), and (3) one gets

$$
\begin{aligned}
& \partial_{t} D_{\mathrm{e}}(\zeta, t)=\partial_{\zeta} v_{\mathrm{e}}(\zeta, t), \\
& \partial_{t} \partial_{\zeta} v_{\mathrm{e}}(\zeta, t)=1-D_{\mathrm{e}}(\zeta, t), \\
& D_{\mathrm{e}}(\zeta, 0)=1, \partial_{\zeta} v_{\mathrm{e}}(\zeta, 0)=0 .
\end{aligned}
$$

The equations (14) and (15), with the initial conditions (16), have unique solution, for which

$$
D_{\mathrm{e}}(\zeta, t)=1, \partial_{\zeta} v_{\mathrm{e}}(\zeta, t)=0, \partial_{\zeta} E_{\mathrm{e}}(\zeta, t)=0 .
$$

So, for $\zeta \in\left(0, \zeta_{\mathrm{e}}(t)\right)$, the functions $x_{\mathrm{e}}(\zeta, t)-\zeta$, $v_{\mathrm{e}}(\zeta, t), E_{\mathrm{e}}(\zeta, t)$ do not depend on $\zeta$; in particular,

$$
\begin{aligned}
& x_{\mathrm{e}}(\zeta, t)-\zeta=b-\zeta_{\mathrm{e}}(t)=b_{1}(t), \\
& E_{\mathrm{e}}(\zeta, t)=E(b, t),
\end{aligned}
$$

and from (4), for the interval $x \in\left(b_{1}(t), b\right)$, one gets

$$
\varphi(b, t)-\varphi\left(b_{1}(t), t\right)=-E(b, t)\left[b-b_{1}(t)\right] .
$$

In the interval $x \in\left(b, b_{3}(t)\right)$ electrons moves in vacuum, integration of (12) gives the equality

$$
E_{\mathrm{e}}(\zeta, t)=E(b, t)+\zeta_{\mathrm{e}}(t)-\zeta,
$$

and from (2) and (12) it follows

$$
\partial_{t} \partial_{\zeta} v_{\mathrm{e}}(\zeta, t)=1
$$

The equations $\partial_{t} \partial_{\zeta} \nu_{\mathrm{e}}(\zeta, t)=-\partial_{\zeta} E_{\mathrm{e}}(\zeta, t)$ and

$$
\partial_{t} D_{\mathrm{e}}(\zeta, t)=\partial_{\zeta} v_{\mathrm{e}}(\zeta, t)
$$

are valid both at $t \in\left(0, \tau_{\mathrm{e}}(\zeta)\right)$, and at $t>\tau_{\mathrm{e}}(\zeta)$. At $t \in\left(0, \tau_{\mathrm{e}}(\zeta)\right)$ (when the relevant electron moves in the ion space), for the corresponding solution, the equalities (17) hold. Then the integration of (22) and (23) with respect to $t$ at $t>\tau_{\mathrm{e}}(\zeta)$ gives

$$
\begin{aligned}
& \partial_{\zeta} v_{\mathrm{e}}(\zeta, t)=t-\tau_{\mathrm{e}}(\zeta), \\
& D_{\mathrm{e}}(\zeta, t)=1+\left[t-\tau_{\mathrm{e}}(\zeta)\right]^{2} / 2 .
\end{aligned}
$$

Using the substitution $x^{\prime}=x_{\mathrm{e}}\left(\zeta_{\mathrm{e}}(\tau), t\right)$ for (4) and integrating by parts, with taking into account (21), (25), and (18), for the interval $x \in\left(b, b_{3}(t)\right)$ one gets

$$
\begin{gathered}
\varphi\left(b_{3}(t), t\right)-\varphi(b, t)=b_{1}(t) B_{1}(t)-B_{2}(t)+ \\
+b_{1}^{2}(t) / 2-E(b, t)\left[b_{3}(t)-b\right],
\end{gathered}
$$

where

$$
B_{j}(t)=\int_{0}^{t} d \tau(t-\tau) b_{1}^{j}(\tau) / j \quad(j=1,2) .
$$

Considering the interval $x \in\left(0, b_{1}(t)\right)$, leaved by electrons, from (4), with use of (6) and (19), one comes to the equalities $E(x, t)=E(b, t)+x-b_{1}(t)$ and

$$
\varphi\left(b_{1}(t), t\right)=-E(b, t) b_{1}(t)+b_{1}^{2}(t) / 2 .
$$

In the interval $x \in\left(b_{3}(t), 1\right)$, the field is uniform, and from (4), with use of (9), (21), and (18) one gets

$$
\begin{gathered}
E(x, t)=E\left(b_{3}(t), t\right)=E(b, t)-b_{1}(t), \\
\varphi(1, t)-\varphi\left(b_{3}(t), t\right)=\left[b_{1}(t)-E(b, t)\right]\left[1-b_{3}(t)\right] .
\end{gathered}
$$

From the equations (1) and (2) and the initial conditions (3), taking into account (29), one comes to the equation $\partial_{t}^{2}\left[b_{3}(t)-b_{1}(t)\right]=b_{1}(t)$ with the initial conditions $\quad \lim _{t \rightarrow 0} \partial_{t}\left[b_{3}(t)-b_{1}(t)\right]=0, \quad b_{3}(0)=b, \quad$ and $b_{1}(0)=0$, from which it is followed the equality

$$
b_{3}(t)=b_{1}(t)+b+B_{1}(t) \text {. }
$$

Using (20), (26), (28), (30), (5), and (31), one gets

$$
E(b, t)=(1-b) b_{1}(t)-B_{2}(t)-U \text {. }
$$


The equation $\partial_{t}^{2} b_{1}(t)=-E(b, t)$, with the equalities (32), (31), and (27), gives the possibility to obtain the functions $b_{1}(t)$ and $b_{3}(t)$ at the considered initial stage of motion. They also may be obtained with use of (31) and integration of the ordinary differential equations,

$$
\begin{aligned}
& \partial_{t} B_{j}(t)=F_{j}(t), \partial_{t} F_{j}(t)=b_{1}^{j}(t) / j, \\
& \partial_{t}^{2} b_{1}(t)=U+B_{2}(t)-(1-b) b_{1}(t),
\end{aligned}
$$

where $F_{j}(t)=\int_{0}^{t} d \tau b_{1}^{j}(\tau) / j, j=1,2$.

\section{REVERSE MOTION}

If the voltage is sufficiently small then at some time instant $t_{\mathrm{R}}$ the electrons in the ion space stop, $v_{\mathrm{e}}\left(\zeta, t_{\mathrm{R}}\right)=0$ for $\zeta \in\left(0, \zeta_{\mathrm{e}}\left(t_{\mathrm{R}}\right)\right)$, and then they begin the reverse movement. The part of electrons come back to the ion space from the vacuum space, and they are moving in the interval $\left(b_{\mathrm{R}}(t), b\right)$ (see Fig. 1,c), where $b_{\mathrm{R}}(t)$ is coordinate of the electron, which has stopped for a moment just at the boundary $b$, $b_{\mathrm{R}}(t)=x_{\mathrm{e}}\left(\zeta_{\mathrm{e}}\left(t_{\mathrm{R}}\right), t\right)$. The stage considered now is restricted in time by the conditions $t>t_{\mathrm{R}}$ and $v_{\mathrm{e}}\left(\zeta_{\mathrm{e}}(t), t\right)<0$, where the designation $\zeta_{\mathrm{e}}(\tau)$ is extended on the electron passing the boundary $x=b$ in any direction. Also, this stage is restricted in time by the assumption that relative disposition of electrons in space is not violated, so that the inequality $D_{\mathrm{e}}(\zeta, t)>0$ is held, for any $\zeta$. Let $\tau_{\mathrm{eR}}(\zeta)$ be the time, when the electron with the initial coordinate $\zeta$ passes the boundary $b$, coming back to the ion space from vacuum, so that $x_{\mathrm{e}}\left(\zeta, \tau_{\mathrm{eR}}(\zeta)\right)=b$. The designation $\tau_{\mathrm{e}}(\zeta)$ is kept for the time of going out from the ion space. At $t>t_{\mathrm{R}}$, during the considered stage, the relationships $\tau_{\mathrm{e}}\left(\zeta_{\mathrm{e}}(t)\right)<t_{\mathrm{R}}<\tau_{\mathrm{eR}}\left(\zeta_{\mathrm{e}}(t)\right)=t$ take place. The equalities (11) and (12) remain valid. Their integration for $\zeta \in\left(\zeta_{\mathrm{e}}\left(t_{\mathrm{R}}\right), \zeta_{\mathrm{e}}(t)\right)$ gives

$$
E_{\mathrm{e}}(\zeta, t)=E(b, t)+\zeta_{\mathrm{e}}(t)-\zeta+x_{\mathrm{e}}(\zeta, t)-b
$$

and for $\zeta \in\left(\zeta_{\mathrm{e}}(t), b\right)$ gives

$$
E_{\mathrm{e}}(\zeta, t)=E(b, t)+\zeta_{\mathrm{e}}(t)-\zeta .
$$

Using (36) and integrating by parts, one gets

$$
\begin{gathered}
\varphi\left(b_{3}(t), t\right)-\varphi(b, t)=E(b, t)\left[b-b_{3}(t)\right]+ \\
+\left\{1+\left[t-T_{\mathrm{R}}(t)\right]^{2} / 2\right\}\left[b-\zeta_{\mathrm{e}}(t)\right]^{2} / 2+ \\
+\left[b-\zeta_{\mathrm{e}}(t)\right] B_{\mathrm{R} 1}(t)-B_{\mathrm{R} 2}(t) .
\end{gathered}
$$

Here $T_{\mathrm{R}}(t)$ is the time of going out from the ion space of the electron, which comes back to it at the time $t, \quad T_{\mathrm{R}}(t)=\tau_{\mathrm{e}}\left(\zeta_{\mathrm{e}}(t)\right), \quad T_{\mathrm{R}}\left(t_{\mathrm{R}}\right)=t_{\mathrm{R}}, \quad T_{\mathrm{R}}(t) \in\left(0, t_{\mathrm{R}}\right) \quad$ for $t>t_{\mathrm{R}}, \quad B_{\mathrm{R} j}(t)=\left[t-T_{\mathrm{R}}(t)\right] F_{\mathrm{R} j}(t)+B_{j}\left(T_{\mathrm{R}}(t)\right) \quad(j=1,2)$, with $F_{\mathrm{R} j}(t)=F_{j}\left(T_{\mathrm{R}}(t)\right)$, and for the time derivatives one has the equalities

$$
\begin{aligned}
& \partial_{t} B_{\mathrm{R} j}(t)=F_{\mathrm{R} j}(t)+\left[t-T_{\mathrm{R}}(t)\right] \partial_{t} F_{\mathrm{R} j}(t), \\
& \partial_{t} F_{\mathrm{R} j}(t)=j^{-1} b_{1}^{j}\left(T_{\mathrm{R}}(t)\right) \partial_{t} T_{\mathrm{R}}(t) .
\end{aligned}
$$

Using (35) and integrating by parts, one gets

$$
\begin{gathered}
\varphi(b, t)-\varphi\left(b_{\mathrm{R}}(t), t\right)=\left[b-b_{\mathrm{R}}(t)\right]^{2} / 2+ \\
+\left[\zeta_{\mathrm{e}}\left(t_{\mathrm{R}}\right)-\zeta_{\mathrm{e}}(t)-E(b, t)\right]\left[b-b_{\mathrm{R}}(t)\right]+S_{\mathrm{R}}(t),
\end{gathered}
$$

where $S_{\mathrm{R}}(t)=\int d \zeta\left[b-x_{\mathrm{e}}(\zeta, t)\right]$, with integral over $\zeta \in\left(\zeta_{\mathrm{e}}\left(t_{\mathrm{R}}\right), \zeta_{\mathrm{e}}(t)\right)$. The integration of the equations (1) and (2) with $E_{\mathrm{e}}(\zeta, t)$ from (35) gives

$$
\begin{gathered}
x_{\mathrm{e}}(\zeta, t)-b=v_{\mathrm{e}}\left(\zeta, \tau_{\mathrm{eR}}(\zeta)\right) \sin \left(t-\tau_{\mathrm{eR}}(\zeta)\right)+ \\
+\int d \tau \sin (t-\tau)\left[\zeta-\zeta_{\mathrm{e}}(\tau)-E(b, \tau)\right],
\end{gathered}
$$

where integral is taken over $\tau \in\left(\tau_{\mathrm{eR}}(\zeta), t\right)$. Substituting $\zeta=\zeta_{\mathrm{e}}(\tau)$ into the definition of $S_{\mathrm{R}}(t)$ and integrating by parts, one gets

$$
\begin{gathered}
S_{\mathrm{R}}(t)=H_{\mathrm{c}}(t) \sin \left(t-t_{\mathrm{R}}\right)-H_{\mathrm{s}}(t) \cos \left(t-t_{\mathrm{R}}\right), \text { where } \\
H_{\mathrm{s}}(t)=\int d \tau \sin \left(\tau-t_{\mathrm{R}}\right) H(\tau), \\
H_{\mathrm{c}}(t)=\int d \tau \cos \left(\tau-t_{\mathrm{R}}\right) H(\tau), \\
H(t)=\left\{1+\left[t-T_{\mathrm{R}}(t)\right]^{2} / 2\right\}^{-1}\left[v_{\mathrm{e}}\left(\zeta_{\mathrm{e}}(t), t\right)\right]^{2}+ \\
+E(b, t)\left[\zeta_{\mathrm{e}}(t)-\zeta_{\mathrm{e}}\left(t_{\mathrm{R}}\right)\right]+\left[\zeta_{\mathrm{e}}(t)-\zeta_{\mathrm{e}}\left(t_{\mathrm{R}}\right)\right]^{2} / 2
\end{gathered}
$$

with integrals in (41), (42) taken over $\tau \in\left(t_{\mathrm{R}}, t\right)$. From (41) and (42), it follows

$$
\begin{aligned}
& \partial_{t} H_{\mathrm{s}}(t)=\sin \left(t-t_{\mathrm{R}}\right) H(t), \\
& \partial_{t} H_{\mathrm{c}}(t)=\cos \left(t-t_{\mathrm{R}}\right) H(t) .
\end{aligned}
$$

Consideration of the intervals $\left(0, b_{1}(t)\right)$, $\left(b_{1}(t), b_{\mathrm{R}}(t)\right)$, and $\left(b_{3}(t), 1\right)$ is similar to consideration of the relevant intervals at the initial stage of motion and, with use of (18), (35), and (36), gives the equalities

$$
\begin{gathered}
\varphi\left(b_{1}(t), t\right)=-E\left(b_{1}(t), t\right) b_{1}(t)+b_{1}^{2}(t) / 2, \\
\varphi\left(b_{\mathrm{R}}(t), t\right)-\varphi\left(b_{1}(t), t\right)=-E\left(b_{\mathrm{R}}(t), t\right)\left[b_{\mathrm{R}}(t)-b_{1}(t)\right], \\
\varphi(1, t)-\varphi\left(b_{3}(t), t\right)=-E\left(b_{3}(t), t\right)\left[1-b_{3}(t)\right], \\
b_{\mathrm{R}}(t)=\zeta_{\mathrm{e}}\left(t_{\mathrm{R}}\right)+b_{1}(t), \\
E\left(b_{\mathrm{R}}(t), t\right)=E\left(b_{1}(t), t\right)=E(0, t)+b_{1}(t), \\
E\left(b_{1}(t), t\right)=E(b, t)+\zeta_{\mathrm{e}}(t)+b_{1}(t)-b,
\end{gathered}
$$

$E\left(b_{3}(t), t\right)=E_{\mathrm{e}}(b, t)=E(b, t)+\zeta_{\mathrm{e}}(t)-b=E(0, t)$.

Substitution of the sum of potential differences over the different intervals into (5) gives the equality

$$
\begin{gathered}
E(b, t)=\left[b-b_{\mathrm{R}}(t)\right]^{2} / 2+b_{1}^{2}(t) / 2-b_{1}(t) b_{\mathrm{R}}(t)+ \\
+\left[b-b_{\mathrm{R}}(t)\right]\left[\zeta_{\mathrm{e}}\left(t_{\mathrm{R}}\right)-\zeta_{\mathrm{e}}(t)\right]-B_{\mathrm{R} 2}(t)+S_{\mathrm{R}}(t)+ \\
+\left[b-\zeta_{\mathrm{e}}(t)\right]\left[B_{\mathrm{R} 1}(t)+1+b_{\mathrm{R}}(t)-b_{3}(t)\right]+ \\
+\left\{1+\left[t-T_{\mathrm{R}}(t)\right]^{2} / 2\right\}\left[b-\zeta_{\mathrm{e}}(t)\right]^{2} / 2-U .
\end{gathered}
$$

The determination of the functions $b_{1}(t)$ and $b_{3}(t)$ may be reduced to the solving of the ordinary differential equations for a few quantities (in addition to $b_{1}(t)$ and $\left.b_{3}(t)\right)$, which are used in the strength calculations (with aid of (47), (48), and (49)) for the equations $\partial_{t}^{2} b_{1}(t)=-E\left(b_{1}(t), t\right)$ and $\partial_{t}^{2} b_{3}(t)=-E\left(b_{3}(t), t\right)$. Some of them (with the references to the equalities for their derivatives, above and below) are the following: $B_{\mathrm{R} j}(t)$ (38), $F_{\mathrm{R} j}(t)(39), H_{\mathrm{s}}(t)(43), H_{\mathrm{c}}(t)(44), \zeta_{\mathrm{e}}(t)(50)$, $T_{\mathrm{R}}(t)(51), v_{\mathrm{e}}\left(\zeta_{\mathrm{e}}(t), t\right)(52), v_{\mathrm{e}}\left(\zeta_{\mathrm{e}}(t), T_{\mathrm{R}}(t)\right)$ (53). Use of the equality (25), which is valid at 
$t \in\left(\tau_{\mathrm{e}}(\zeta), \tau_{\mathrm{eR}}(\zeta)\right)$, and taking of the time derivative of the equality $x_{\mathrm{e}}\left(\zeta_{\mathrm{e}}(t), t\right)=b$ at $t>t_{\mathrm{R}}$ gives

$$
\partial_{t} \zeta_{\mathrm{e}}(t)=-\left\{1+\left[t-T_{\mathrm{R}}(t)\right]^{2} / 2\right\}^{-1} v_{\mathrm{e}}\left(\zeta_{\mathrm{e}}(t), t\right),
$$

$\partial_{t} \zeta_{\mathrm{e}}(t)>0$, at the considered stage. Differentiation of the equality $\zeta_{\mathrm{e}}\left(\tau_{\mathrm{e}}(\zeta)\right)=\zeta$ with use of (50) gives $\partial_{\zeta} \tau_{\mathrm{e}}(\zeta)=-\left[v_{\mathrm{e}}\left(\zeta, \tau_{\mathrm{e}}(\zeta)\right)\right]^{-1}$, and for $t>t_{\mathrm{R}}$ one gets

$$
\partial_{t} T_{\mathrm{R}}(t)=-\left[v_{\mathrm{e}}\left(\zeta_{\mathrm{e}}(t), T_{\mathrm{R}}(t)\right)\right]^{-1} \partial_{t} \zeta_{\mathrm{e}}(t) .
$$

Taking into account the equality (24), which is valid at $t \in\left(\tau_{\mathrm{e}}(\zeta), \tau_{\mathrm{eR}}(\zeta)\right)$, for $t>t_{\mathrm{R}}$ one gets

$$
\begin{aligned}
& \partial_{t} v_{\mathrm{e}}\left(\zeta_{\mathrm{e}}(t), t\right)=\left[t-T_{\mathrm{R}}(t)\right] \partial_{t} \zeta_{\mathrm{e}}(t)-E(b, t), \\
& \partial_{t} v_{\mathrm{e}}\left(\zeta_{\mathrm{e}}(t), T_{\mathrm{R}}(t)\right)=-E\left(b, T_{\mathrm{R}}(t)\right) \partial_{t} T_{\mathrm{R}}(t) .
\end{aligned}
$$

The quantity $b_{\mathrm{R}}(t)$ is given by (46). As $T_{\mathrm{R}}(t) \in\left(0, t_{\mathrm{R}}\right)$, the quantities $v_{\mathrm{e}}\left(\zeta_{\mathrm{e}}(t), T_{\mathrm{R}}(t)\right)$ and $E\left(b, T_{\mathrm{R}}(t)\right)$ are related to the initial stage of motion, which has been described above. The characteristics of that stage may be obtained with integration of the equations (33), (34) all over again (in opposite time direction, as $\partial_{t} T_{\mathrm{R}}(t)<0$, for $\left.t>t_{\mathrm{R}}\right)$, along with the integration of the equations for the mentioned few quantities related to the considered reverse motion stage.

The equations for the field strength determination written in this section are valid only if the relative disposition of electrons in space is not violated, so that the inequality $D_{\mathrm{e}}(\zeta, t)>0$ is held. The conditions, which give the time of the inequality violation, may be got from (40). For $\left\{\zeta \in\left(\zeta_{\mathrm{e}}\left(t_{\mathrm{R}}\right), \zeta_{\mathrm{e}}(t)\right), t>t_{\mathrm{R}}\right\}$, using (24) and (25), one comes to the equalities

$$
\begin{gathered}
\partial_{\zeta} v_{\mathrm{e}}\left(\zeta, \tau_{\mathrm{eR}}(\zeta)\right)= \\
=\tau_{\mathrm{eR}}(\zeta)-\tau_{\mathrm{e}}(\zeta)-E\left(b, \tau_{\mathrm{eR}}(\zeta)\right) \partial_{\zeta} \tau_{\mathrm{eR}}(\zeta) \\
v_{\mathrm{e}}\left(\zeta, \tau_{\mathrm{eR}}(\zeta)\right) \partial_{\zeta} \tau_{\mathrm{eR}}(\zeta)= \\
=\partial_{\zeta} x_{\mathrm{e}}\left(\zeta, \tau_{\mathrm{eR}}(\zeta)\right)-D_{\mathrm{e}}\left(\zeta, \tau_{\mathrm{eR}}(\zeta)\right)= \\
=-1-\left[\tau_{\mathrm{eR}}(\zeta)-\tau_{\mathrm{e}}(\zeta)\right]^{2} / 2
\end{gathered}
$$

and with taking them into account, from (40) one gets

$$
D_{\mathrm{e}}(\zeta, t)=1+A_{\mathrm{e}}(\zeta) \cos \left[t-\tau_{\mathrm{eR}}(\zeta)-\Phi_{\mathrm{e}}(\zeta)\right],
$$

where $\Phi_{\mathrm{e}}(\zeta)=\arctan \left\{2 /\left[\tau_{\mathrm{eR}}(\zeta)-\tau_{\mathrm{e}}(\zeta)\right]\right\}$, $A_{\mathrm{e}}(\zeta)=2 \cos \Phi_{\mathrm{e}}(\zeta) / \sin ^{2} \Phi_{\mathrm{e}}(\zeta)$, so that $A_{\mathrm{e}}(\zeta)>0$, $\Phi_{\mathrm{e}}(\zeta) \in(0, \pi / 2), \quad \partial_{\zeta} A_{\mathrm{e}}(\zeta)>0, \quad \partial_{\zeta} \Phi_{\mathrm{e}}(\zeta)<0$. As it follows from (54), if $A_{\mathrm{e}}(\zeta)<1$ then $D_{\mathrm{e}}(\zeta, t)>0$, for any $t$. But if $A_{\mathrm{e}}\left(\zeta_{\mathrm{e}}(\tau)\right)>1$, for some $\tau>t_{\mathrm{R}}$, then there is such $t$, for which the inequality $D_{\mathrm{e}}\left(\zeta_{\mathrm{e}}(\tau), t\right)<0$ takes place, that is, the order of electrons disposition is violated, near the electron with the initial coordinate $\zeta_{\mathrm{e}}(\tau)$, which comes back to the ion space at the time $\tau$. The violation of the electron relative disposition is connected with their decreased density when they come back to the ion space, and with the consequent excess of positive charge in the interval $\left(b_{\mathrm{R}}(t), b\right)$, which causes some acceleration of electrons towards one another.

\section{MOTION WITH INTERMEDIATE GAP}

If $b<1 / 2$ and the applied voltage is sufficiently large then the initial stage of the process, which has ISSN 1562-6016. BAHT. 2019. №6(124) been considered above, is followed by going out of all electrons from the ion space with gap appearing between the ion and electron spaces (see Fig. 1,d). Let $t_{\mathrm{G}}$ be the time of transition to this stage, so that $x_{\mathrm{e}}\left(0, t_{\mathrm{G}}\right)=b, \zeta_{\mathrm{e}}\left(t_{\mathrm{G}}\right)=0$. At $t>t_{\mathrm{G}}$ all electrons are situated in the interval $\left(b_{1}(t), b_{3}(t)\right)$ with $b_{1}(t)>b$. The field strength boundary values obey to the equalities

$$
E\left(b_{3}(t), t\right)+b=E(b, t)=E\left(b_{1}(t), t\right) .
$$

Consideration of the intervals $\left(b_{3}(t), 1\right)$, $\left(b_{1}(t), b_{3}(t)\right),\left(b, b_{1}(t)\right)$, and $(0, b)$ gives (45) and the equalities

$$
\begin{gathered}
\varphi\left(b_{3}(t), t\right)-\varphi\left(b_{1}(t), t\right)=E(b, t)\left[b_{1}(t)-b_{3}(t)\right]+ \\
+\left(t-t_{\mathrm{G}}\right)\left[b F_{1}\left(t_{\mathrm{G}}\right)-F_{2}\left(t_{\mathrm{G}}\right)\right]+b B_{1}\left(t_{\mathrm{G}}\right)-B_{2}\left(t_{\mathrm{G}}\right)+ \\
+\left[2+\left(t-t_{\mathrm{G}}\right)^{2}\right] b^{2} / 4 \\
\varphi\left(b_{1}(t), t\right)-\varphi(b, t)=-E(b, t)\left[b_{1}(t)-b\right], \\
\varphi(b, t)=-E(b, t) b+b^{2} / 2 .
\end{gathered}
$$

Their substitution into (5) gives the equality

$$
\begin{aligned}
& E(b, t)=b^{2}\left[1+\left(t-t_{\mathrm{G}}\right)^{2} / 4\right]+b\left[1-b_{3}(t)\right]-U+ \\
& +\left(t-t_{\mathrm{G}}\right)\left[b F_{1}\left(t_{\mathrm{G}}\right)-F_{2}\left(t_{\mathrm{G}}\right)\right]+b B_{1}\left(t_{\mathrm{G}}\right)-B_{2}\left(t_{\mathrm{G}}\right),
\end{aligned}
$$

use of which on the integration of the equations $\partial_{t}^{2} b_{1}(t)=-E(b, t)$ and $\partial_{t}^{2} b_{3}(t)=-E(b, t)+b$ gives the functions $b_{1}(t)$ and $b_{3}(t)$.

If $b<1 / 2$ and the applied voltage is not too large then the gap may disappear, with coming back to the ion space by the part of electrons. Let $t_{\mathrm{B}}$ be the time of the first electron arriving at the boundary $b$ in the opposite direction, so that $t_{\mathrm{B}}>t_{\mathrm{G}}, x_{\mathrm{e}}\left(0, t_{\mathrm{B}}\right)=b, \quad \zeta_{\mathrm{e}}\left(t_{\mathrm{B}}\right)=0$, $v_{\mathrm{e}}\left(0, t_{\mathrm{B}}\right)<0$. To describe the electron motion at $t>t_{\mathrm{B}}$, one may take the equalities obtained above for such reverse motion, which arises without the intermediate gap appearing, put $b_{\mathrm{R}}(t)=b_{1}(t)$, and replace $t_{\mathrm{R}}$ with $t_{\mathrm{B}}$ or $t_{\mathrm{G}}$, in the relevant places (in particular, there should be $\tau_{\mathrm{e}}\left(\zeta_{\mathrm{e}}\left(t_{\mathrm{B}}\right)\right)=t_{\mathrm{G}}$, instead of $\left.\tau_{\mathrm{e}}\left(\zeta_{\mathrm{e}}\left(t_{\mathrm{R}}\right)\right)=t_{\mathrm{R}}\right)$.

The both considered types of the reverse motion stages are restricted in time by violation of the conditions $v_{\mathrm{e}}\left(\zeta_{\mathrm{e}}(t), t\right)<0$ or $D_{\mathrm{e}}(\zeta, t)>0$ (at any $\zeta$ ). The calculations for the electron motion after the violation were carried out with use of one-dimensional mesh along the initial electron coordinate and with use of the equation (13) for the field strength determination.

\section{RESULTS}

The calculations for electron motion during the time up to the time $t_{3}$ of the first electron arriving at anode (so that $b_{3}\left(t_{3}\right)=1$ ) were carried out.

In the Fig. 2 the dependences of the ratio $\eta$ of the attained energy and one corresponding to voltage on the voltage $U$ for the different values of the relative width $b$ of the ion space are shown. It is also used the quantity $\bar{U}=U / \delta^{3}$, where $\delta=1-b$. The dashed curve is related to the limit, when $b \rightarrow 1$, but the value of $\bar{U}$ is kept constant, and $U$ is accordingly decreases. 
To get the equations for this limit case, it should be taken into account that if the relatively small charge is moving in the ionized medium then the corresponding field perturbation is dispersed during the time reciprocal to the plasma frequency and at the distance equal to the product of the mentioned time and the charge velocity. That is, the voltage decrease and the consequent decrease of the velocity of the electrons, which come back to the ion space, leads to vanishing of the perturbation of the potential difference over the ion space caused by these electrons, and so, the field strength in the vacuum space (and the electron motion there) become independent on the details of the electron motion in the ion space.

From the equations (31), (33), and (34), taking $\tau=t \delta^{1 / 2}, \quad \bar{b}_{3}(\tau)=\left[b_{3}(t)-b\right] / \delta, \quad \bar{B}_{2}(\tau)=B_{2}(t) / \delta^{3}$, $\bar{B}_{1}(\tau)=B_{1}(t) / \delta$, and $\bar{b}_{1}(\tau)=b_{1}(t) / \delta^{2}$, after the limit transition $b \rightarrow 1$ one gets the equality $\bar{B}_{1}(\tau)=\bar{b}_{3}(\tau)$ and the equations $\partial_{\tau}^{2} \bar{b}_{3}(\tau)=\bar{b}_{1}(\tau), \partial_{\tau}^{2} \bar{B}_{2}(\tau)=\bar{b}_{1}^{2}(\tau) / 2$, and $\partial_{\tau}^{2} \bar{b}_{1}(\tau)=\bar{U}+\bar{B}_{2}(\tau)-\bar{b}_{1}(\tau)$, integration of which gives the mentioned dashed curve. The calculations give the value of $\eta$ near to 1.87475 for $\bar{U}$ near to 0.165 .
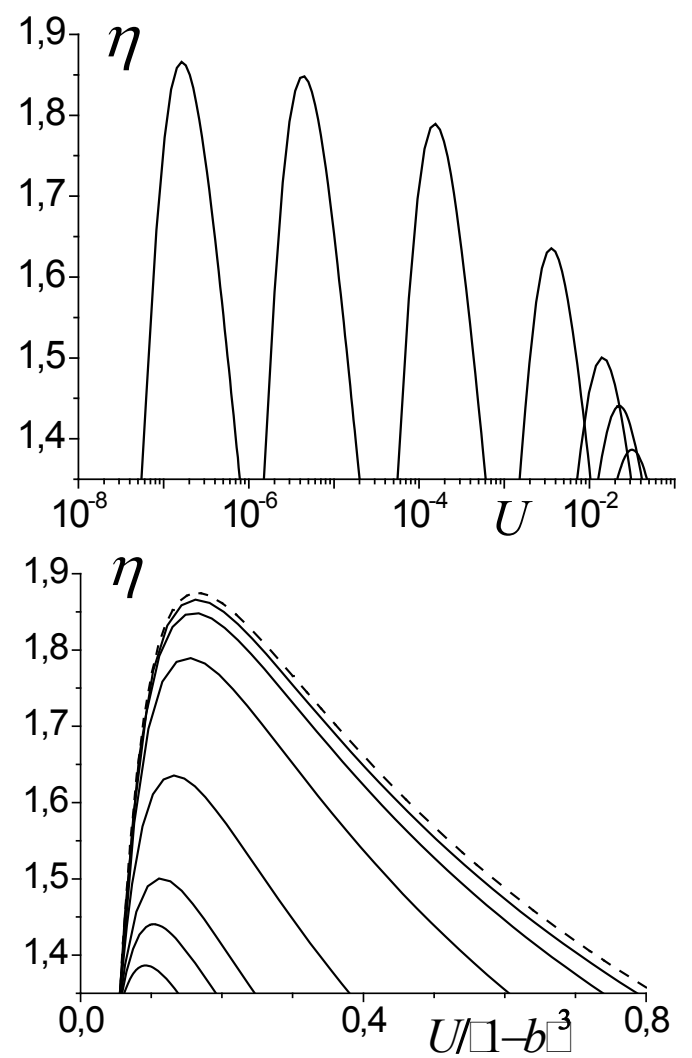

Fig. 2. Dependence of the coefficient of energy increase $\eta$ on the voltage $U$ for different values of relative

width $b$ of ion space $(0.3,0.4,0.5,0.7,0.9,0.97,0.99$, in ascending order for $\eta$ maximums; the dashed curve $i$ s related to the limit $b \rightarrow 1$ )

The Fig. 3, in the different scales, gives the ranges of the voltage $U$ values, for the given value of the relative width $b$ of the ion space, which correspond to presence or absence of the considered above electron motion stages during the time $t \in\left(0, t_{3}\right)$. The points $\mathrm{O}, \mathrm{D}$, and $\mathrm{F}$ on the plane $(b, U)$ have the coordinates $(0,0),(1,0)$, and $(1 / 2, \infty)$, the points $A, B, C$, and $E$ are near to the points $\quad(0.01841,0.01353), \quad(0.01854,0.01352)$, $(0.06732,0.03117)$, and $(0.2329,0.08567)$, respectively.

The highest of the curves, connecting the points $\mathrm{A}$ and $\mathrm{E}$, which is designated $\{\mathrm{AE}\}^{+}$below, and the curve ED give the voltage values for the beginning of the reverse motion just at the time $t_{3}$, after the intermediate gap appearing $\left(\{\mathrm{AE}\}^{+}\right)$or in the process without its appearing (ED). The next (in descending order) of the curves, connecting the points $\mathrm{A}$ and $\mathrm{E}$, which is designated $\{\mathrm{AE}\}^{-}$below, corresponds to the intermediate gap disappearing (after the preceding appearing) just at the time $t_{3}$.
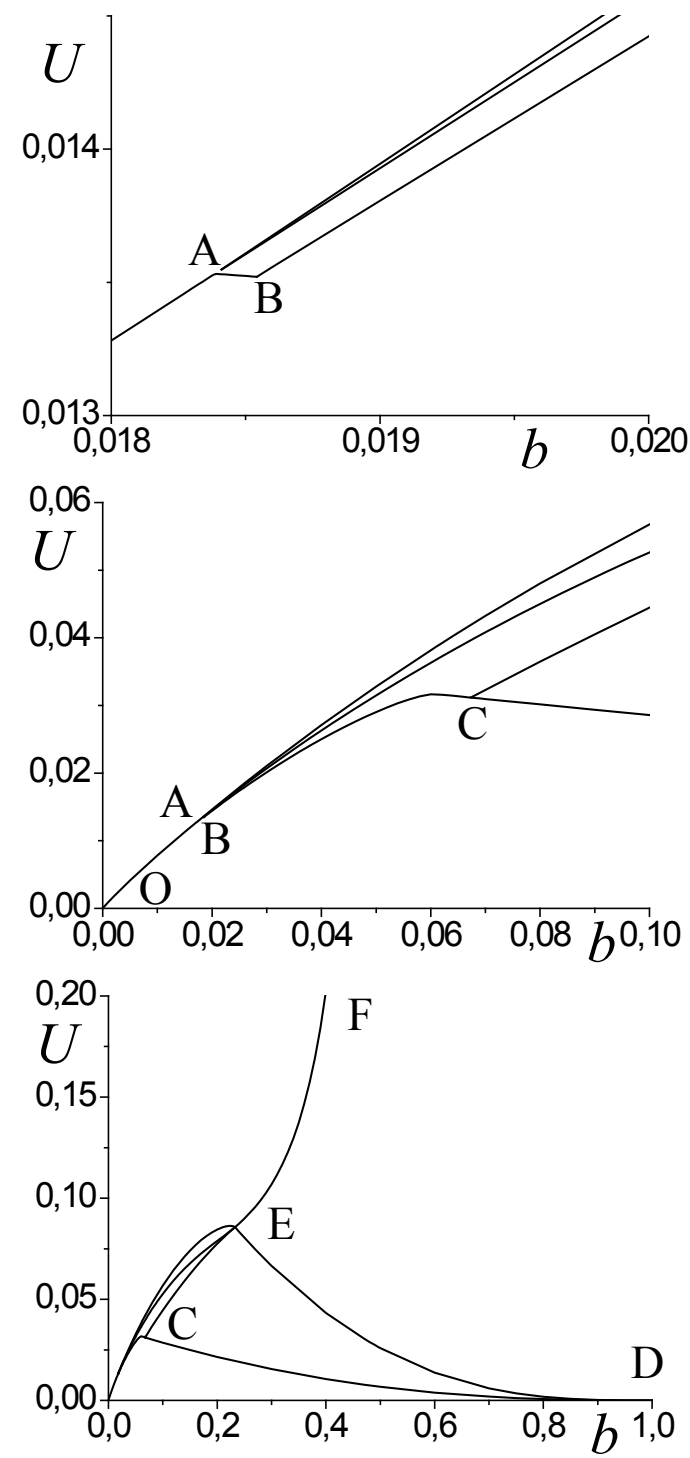

Fig. 3. The areas on the $(b, U)$ plane, related to presence of the different motion stages (in text)

The curve EF gives the voltage values, at which the initial stage of motion continues up to the time $t_{3}$ ending by the gap appearing. The curve CE corresponds to stopping of the electron space boundary $b_{1}(t)$ just at the ion space boundary $b$ (before the following reverse motion beginning), so that $\left\{b_{1}(t)=b, \partial_{t} b_{1}(t)=0\right\}$ at $t=t_{\mathrm{R}} \in\left(0, t_{3}\right)$. The curves $\mathrm{BC}$ and $\mathrm{CD}$ correspond to the violation of electron order just at the time $t_{3}$, after 
the preceding gap appearing and disappearing (BC) or in the process without gap appearing (CD). The curve $\mathrm{OA}$ corresponds to the gap appearing, and later, at some $t \in\left(0, t_{3}\right)$, the gap disappearing for a moment, so that $\left\{b_{1}(t)=b, \partial_{t} b_{1}(t)=0\right\}$ at this $t$; for the point A such disappearing occurs at the time $t_{3}$. For the voltage values at the curve $A B$, the second change of the motion direction at the boundary $b$ (after the gap appearing and disappearing) occurs at the time $t_{3}$.

The area on the plane $(b, U)$ between the curves $\{\mathrm{AE}\}^{+}$and $\{\mathrm{AE}\}^{-}$corresponds to the case when the reverse motion begins after the intermediate gap appearing and, at least, up to the time $t_{3}$ the gap does not disappear. The area bounded by the curves $\mathrm{AB}, \mathrm{BC}, \mathrm{CE}$, and $\{\mathrm{AE}\}^{-}$corresponds to the case when the reverse motion begins after the gap appearing, later, at some $t \in\left(0, t_{3}\right)$, the gap disappears, but, at least, up to the time $t_{3}$ there are neither the second change of the motion direction at the boundary $b$, no the violation of electron order. The area bounded by the curves $\mathrm{CE}, \mathrm{CD}$, and DE corresponds to the case when the reverse motion happens without gap appearing and the electron order does not violate. The area below the curves OA, $\mathrm{AB}, \mathrm{BC}$, and $\mathrm{CD}$ corresponds to the case when at some $t \in\left(0, t_{3}\right)$ the process comes either to the second change of the motion direction at the boundary $b$, or to the electron order violation. The area above the curves OA, $\{\mathrm{AE}\}^{+}$, and EF corresponds to the motion of all electrons without change of direction and with the gap appearing at some $t \in\left(0, t_{3}\right)$. The area on the right of the curves DE and EF corresponds to continuing of the initial stage of motion, at least, up to the time $t_{3}$.

Some change of the model gives the limit value 2 for the ratio $\eta$. Namely, let electrons become immobilized when stop in the ion space (for example, through intensive recombination). Then, in the case of $1-b<<1$ with application of a very small voltage $U$, the field strength in the ion space after the electron stopping is near to $-U$ (as the acceleration-deceleration process is approximately symmetric, due to the dependence of the strength on the $b_{1}(t)$ value near to linear one), and so, the potential difference over the vacuum space is near to $2 U$. And the part of the electrons having gone out of the ion space are accelerated by the field strength near to $2 \bar{U} \delta^{2}$ giving the $\eta$ value near to 2 . In the model considered above, electrons do not become immobilized when stopping in the ion space. Their movement to cathode leads to decrease of the field strength in the point $b_{3}(t)$, and to decrease of the attainable $\eta$ value.

\section{CONCLUSIONS}

In absence of the external circuit the movement of electrons to anode leads to decrease of the potential difference between electrodes. The keeping up of the potential difference through the external circuit leads to the field strength increase in the leading point of electron flow, and so, to the increase of the final energy of some electrons (at their going out to anode), in comparing with one corresponding to the given potential difference. For the diode with the near cathode layer of the immobilized ions compensated by electrons at the start, the maximum value of the coefficient of the mentioned energy increase is near to 1.87475 .

\section{REFERENCES}

1. A.V. Pashchenko, I. A. Pashchenko. Non-linear selfacceleration of electrons emitted by plasma cathode // Problems of Atomic Science and Technology. Series "Nuclear Physics Investigations”. 2016, № 5, p. 114-117.

Article received 30.09.2019

\section{ЭНЕРГИЯ ЭЛЕКТРОНОВ, ДОСТИЖИМАЯ В ДИОДЕ С ПЛАЗМЕННЫМ КАТОДОМ ПРИ ДАННОМ НАПРЯЖЕНИИ \\ В. Остроушко, А. Пащенко, И. Пащенко}

Рассмотрено ускорение электронов в одномерном промежутке, часть которого заполняют ионы, в начальный момент компенсированные электронами. Для стадий прямого и обратного движения части электронов задача сведена к численному решению обыкновенных дифференциальных уравнений для некоторой совокупности величин, зависящих от времени. Отношение достижимой энергии к энергии, соответствующей напряжению, максимально, и близко к 1,87475 для относительно малых значений ширины пространства без ионов и при применении определенного напряжения, которое при уменьшении этой ширины надо уменьшать как куб ширины.

\section{ЕНЕРГІЯ ЕЛЕКТРОНІВ, ДОСЯЖНА В ДІОДІ 3 ПЛАЗМОВИМ КАТОДОМ ПРИ ДАНІЙ НАПРУЗІ}

\section{В. Остроуико, А. Пащенко, І. Пащенко}

Розглянуто прискорення електронів в одновимірному проміжку, частину якого заповнюють іони, у початковий момент компенсовані електронами. Для стадій прямого та зворотного руху частини електронів задачу зведено до числового розв'язання звичайних диференційних рівнянь для певної сукупності величин, залежних від часу. Відношення досяжної енергії до енергії, що відповідна напрузі, максимальне, та близьке до 1,87475 для відносно малих значень ширини простору без іонів та при застосуванні певної напруги, яку при зменшенні тієї ширини треба зменшувати як куб ширини. 